\title{
Nachschlagen von Arzneimittel-Fachinformation
}

Neuner-Jehle, S

Posted at the Zurich Open Repository and Archive, University of Zurich ZORA URL: https://doi.org/10.5167/uzh-93658

Journal Article

Published Version

Originally published at:

Neuner-Jehle, S (2013). Nachschlagen von Arzneimittel-Fachinformation. PrimaryCare, 13(8):136. 


\title{
Nachschlagen von Arzneimittel- Fachinformation
}

\author{
Ein Beitrag aus der Reihe «Die Werkzeugkiste des Familienarztes»
}

Vor Jahrhunderten erforderte das Nachschlagen von detaillierten Informationen zu Arzneimitteln gut trainierte Bizepsmuskeln: Die einzigen Quellen waren ledergebundene Pharmakopöen, eigentliche «Arzneimittelschätze» im Besitz von Apothekern, Alchimisten und Ärzten. Auch das 2013 zum letzten Mal in Papierform aufgelegte «Arzneimittelkompendium der Schweiz ${ }^{\odot}$ » hat jedes Jahr an Volumen zugenommen und lastet schwer im Sprechstundenregal. Wie kommt man heute, im Zeitalter der Online-Suchmaschinen, rasch zu verlässlicher Information?

\section{www.compendium.ch}

Die Firma Documed (Galenica-Gruppe), welche das oben erwähnte Arzneimittelkompendium herausgab, bietet weiterhin eine nützliche Übersicht über die zugelassenen Medikamente mit Suchfunktion. Neben der üblichen Suche nach Name, Wirkstoff, Firma oder Freitext bestehen diverse Abfragemöglichkeiten wie Interaktionen, Identa, Therapeutisches Register oder Updates. Die aktuellen kommerziellen Daten wie Preise, Kassenzulässigkeit mit Limitatio sind den Packungen zugeordnet. Bis dato ist der Zugang frei, für einen späteren Zeitpunkt wird die Anmeldung via Swiss-RxLogin angekündigt.

Der grosse Wermutstropfen: Nach einem Entscheid des Bundesverwaltungsgerichts von 2011 müssen Zulassungsinhaberinnen die Fach- und Patienteninformationen nicht mehr bei einem privaten Anbieter publizieren. Die Vollständigkeit bisheriger Verzeichnisse dürfte dadurch leiden und damit auch die Arzneimittelsicherheit.

\section{www.swissmedicinfo.ch}

Swissmedic ist nun in diese Bresche gesprungen und bietet unter obiger Webadresse ein «Arzneimittel Informations- und Publikationssystem» (AIPS) an [1]. Dort lassen sich die offiziellen Fach- und Patienteninformationen nach Präparatename, Wirkstoff, ATCCode, Zulassungsinhaberin oder Volltext abfragen. Ausserdem gibt es je eine Liste von neuen (letzte 3 Monate) und geänderten (letzte 4 Wochen)Texten.

\section{www.kinderdosierungen.ch}

Für spezielle, sprich pädiatrische Bedürfnisse führt das Kinderspital Zürich eine neue Website, weil viele bei Kindern eingesetzte Heilmittel bezüglich Wirksamkeit, Dosierung und Nebenwirkungen ungenügend untersucht und damit in gängigen Verzeichnissen ungenügend abgebildet sind [2]. Fürs erste Log-in (Swiss-RxLogin) benötigt man seine GLN (Global Location Number), welche die integrierte Suchmaschine aber nach Angaben der professionellen Personalien liefert. Nützlich auf der Website ist ein Dosierungsrechner für Alter, Gewicht und Grösse der kleinen Patienten.

\section{medkalender}

Der «medkalender» aus dem EMH-Verlag hat eine beachtliche Tradition (erscheint seit 1879, damals als «Medicinalkalender für die Schweiz») und bietet eine gewichtete Fülle an Informationen über Arzneimittel im Kitteltaschenformat, nach Fachgebieten,
Wirkstoffgruppen, Wirksubstanzen, Originalpräparaten und Generika geordnet. Au-

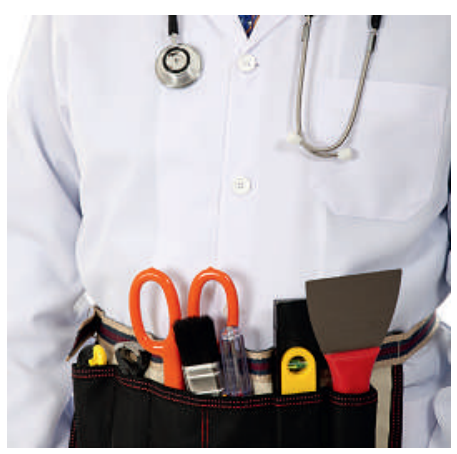
sserdem fliessen nützliche Kommentare aus der «pharma-kritik» ein - der Internist und klinische Pharmakologe Etzel Gysling firmiert bei beiden Produkten als (Mit-)Herausgeber.

Ebenfalls aus seiner Feder stammt das beliebte Werk:

\section{«Hundert wichtige Medikamente»}

Letztmals 2005 im Infomed-Verlag aufgelegt.

Ein Klick auf die Webseite des Verlags (www.infomed.ch) lohnt sich auch anderweitig, zum Beispiel wegen der spannenden und aktuellen Rubrik «Bad Drug News».

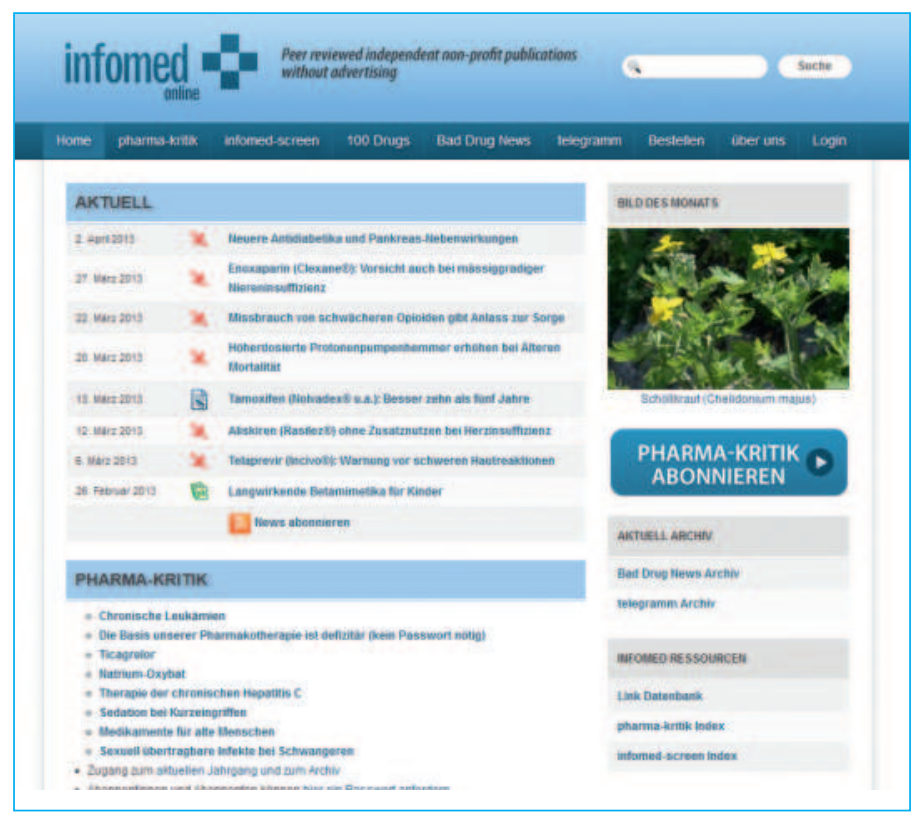

Literatur

1 Mathys Badertscher K. Publikation aller Fachinformationen in einem Verzeichnis. Schweizerische Ärztezeitung 2013;94(3):52

2 Glanzmann C, Vonbach P. www.kinderdosierungen.ch: Neue Website mit Informationen zur Medikamentengabe bei Kindern. Schweiz Med Forum 2012;12(50):985

\section{Korrespondenz:}

Dr. med. Stefan Neuner-Jehle, MPH

Institut für Hausarztmedizin Zürich

Pestalozzistrasse 24

8091 Zürich

sneuner[at]bluewin.ch 